disappeared, leaving one or two longish ones. The colour was mostly white, but sometimes reddish in parts, especially nearly due north.

"A curious feature was an oblique band of light, which came and went across near the summits of the vertical beams. I do not think this was a belt of illuminated cirrus, as its brightness seemed to vary independently of the vertical beams, but it is possible it may have been. The lights had diminished considerably by about 3.45 a.m., but had brightened again, though slightly, when. I looked out a few minutes later. I do not know what time the display ended."

Stonyhurst College Observatory, March 29.

\section{Some Methods of Approximate Integration and of Computing Areas.}

The formulæ which Mr. Percival gives in Nature for March i8 for approximate integration are well known, but there are one or two points in connection with them which are frequently overlooked, especially by writers of books on mathematics for engineers.

(I) The areas bounded by curves the equations of which are of the form

$$
y=a+b x+c x^{2}+\ldots+k x^{n}
$$

can be obtained from the values of $2 m+I$ equidistant ordinates, not only when $n=2 m$, but also when $n=2 m+1$. That this is so is seen most easily by taking the origin at the centre of the range of integration and noting that

$$
\int_{-h}^{+h} x^{2 m+1} d x=0
$$

For example, Simpson's first, or three-ordinate, rule gives the area of the cubic

$$
y=a+b x+c x^{2}+d x^{3}
$$

with perfect accuracy, and for this purpose his second, or four-ordinate, rule is in no way superior.

(2) By a very small change in one of the coefficients Weddle threw the seven-ordinate formula (No. 6 in Mr. Percival's letter) into the very convenient form

$$
\mathrm{A}=\frac{3 h}{\mathrm{IO}}\left[y_{1}+y_{3}+y_{5}+y_{7}+5\left(y_{2}+y_{6}\right)+6 y_{4}\right] \text {. }
$$

The loss of accuracy which the change involves is exceedingly small.

(3) Formulæ based upon the assumption that the boundary curve can be represented by an equation of the form above stated give unsatisfactory results when the actual boundary has tangents at right angles to the $x$-axis. This is really the reason why none of the results obtained by Mr. Percival in applying his formulæ to the quadrature of a circle possess a higher degree of accuracy than that represented by the admission of errors of the order of $\mathrm{x}$ per cent.

If we suppose the curve to cut the axis of $x$ at right angles at the origin, it is better to assume that it can be represented by $y=a x^{\frac{1}{2}}+b x$ in the neighbourhood of that point.

If $y_{1}, y_{2}$ be the ordinates at $x=h, x=2 h$, the area bounded by the curve, the axis of $x$ and the ordinate $y_{2}$ is given by

$$
\mathrm{A}=\frac{4 h}{15}\left[4 \sqrt{2 y_{1}+y_{2}}\right]
$$

The much higher degree of accuracy resulting from the employment of this formula may be illustrated by applying it to Mr. Percival's example of the quadrant of a circle.

The seven ordinates are :-

$$
\begin{aligned}
y_{0}=0 & y_{4}=0.9428090 \\
y_{1}=0.5527708 & y_{5}=0.9860133 \\
y_{2}=0.7453560 & y_{6}=1 \\
y_{3}=0.8660254 & \\
& \text { NO. 263 I, VOL. IO5] }
\end{aligned}
$$

Using the above formula to find the area between the ordinates $y_{0}$ and $y_{2}$, and Simpson's first formula for the part between $y_{2}$ and $y_{6}$, we obtain the value 0.785387 I. The true value is 0.7853982 ; hence the percentage error is only 0.0014 , which compares very favourably with the errors ranging from 0.8 to $\mathrm{I} \cdot 34$ per cent. obtained by using the usual formulæ for the whole range.

Mr. Percival's example clearly shows that when the curve has a tangent at right angles to the axis, no material reduction in error is attained by using formulæ with a larger number of ordinates. The use of Simpson's formula over ordinary ranges and of the formula given above in the neighbourhood of such tangents will prove much less laborious and far more accurate.

King's College, Strand, March 22.

J. B. Dale.

In Nature of March I8 Mr. A. S. Percival gives an example (the quadrant of a circle) in which Simpson's rule (sometimes called his first rule) is more accurate than the "three-eighths" rule, and he remarks: "This result is curious, and shows that a small arc of a circle approaches more nearly to a small arc of a parabola than to a small arc of any cubic curve." Permit me to point out that this inference is not valid, and is based on the almost universal illusion that Simpson's rule is correct to the second order only, i.e. for the parabola

$$
y=a+b x+c x^{2} .
$$

It is easy to show by simple integration that Simpson's rule holds to the third order, i.e. for all cubics of the form

$$
y=a+b x+c x^{2}+d x^{3},
$$

passing through the three chosen points. It is thus precisely accurate, not only for the parabola, but also for a singly infinite number of curves passing through the three points, even if an inflexion occurs.

One would therefore expect (which I believe to be the case) that where both rules can be applied (e.g. if there are seven ordinates) Simpson's rule would be more accurate than the "three-eighths" rule, which is precisely true only for a single curve passing through four consecutive points.

In some cases, when the gradient is not rapid, Simpson's rule is highly accurate. Dr. Lamb " Infinitesimal Calculus," p. 278) gives an example in the evalution of $\pi$ to six decimal places from the equation

$$
\int_{0}^{1} \frac{a}{1+x^{2}}=\frac{\pi}{4}
$$

by taking ten equidistant values for $x$, but he does not notice the illusion to which I refer. I am surprised that such a simple and easily tested truth should sollong have escaped the notice of many expert mathematicians.

Trinity College, Dublin, March 20.

\section{Gravitational Deflection of High-speed Particles.}

THE result mentioned by $\mathrm{Mr}$. Leigh Page and verified by Prof. Eddington (NATURE, March II, p. 37), that the gravitational effect on a particle travelling radially is a repulsion if the speed exceeds $I / \sqrt{3}$ times the light-velocity, is given by Hilbert in the Göttinger Nachrichten for 1917. The same paper contains interesting remarks on the path of a particle or lightpulse moving spirally round the gravitation centre.
Hymers College, Hull.
H. G. FORDER. 